Arch. Tierz., Dummerstorf 50 (2007) 5, 455-463

\footnotetext{
${ }^{1}$ Research Institute for Cattle Breeding, Ltd., Rapotín, Vikýřovice, Czech Republic

${ }^{2}$ Mendel University of Agriculture and Forestry Brno, Brno, Czech Republic.

${ }^{3}$ Agroresearch, Ltd., Rapotín, Vikýřovice, Czech Republic
}

\title{
The effects of inbreeding on service period and pregnancy length in Holsteins and Czech Fleckviehs after the first calving
}

\begin{abstract}
The effects of inbreeding level on service period (SP) and pregnancy length (PL) were monitored in Holstein and Czech Fleckvieh after the first calving. The levels of inbreeding $\left(F_{X}\right)$ ranged from 1.25 to $25 \%$. SP and PL of inbred cows were compared with their outbred equals - half-sisters, sharing the same sire $(n=1824)$ and their first calving happened in the same farm and at the same time ( \pm 3 month). Database with the number of 631810 animals (year of calving 1985-2004) was used for analyses. Inbred cows were grouped according to $F_{x}$ coefficient (1.5-2.3 \%; 3.0-5.0 \%; 8.0-12.5 \%; $25 \%$ ). The GLM with fixed effects (the breed, breeding value of the sire and monitored individual for milk production, year of calving, age at first calving) was applied to all data. The effect of inbreeding depression on SP was more pronounced at higher levels of inbreeding $(+2.81$; $+3.35 ;+4.53$; $+8.23 \mathrm{~d}$, respectively according to above mentioned $\mathrm{F}_{\mathrm{x}}$ groups) but non-significant. Differences in PL (+0.3; +0.32; -0.08 and $1.68 \mathrm{~d}$ ) were not significant either. Average value of PL for inbred animals was 0.43 $\mathrm{d}$ higher. The correlation coefficient was +0.023 for SP and +0.0658 for PL. The $\mathrm{F}_{\mathrm{x}}$ coefficient increasing by 1 \% extends the SP by 0.22 day and PL by 0.063 day. There was a higher variability of SP and PL in inbred cows. The length of calving interval between the first and second lactation is affected more by SP than PL.
\end{abstract}

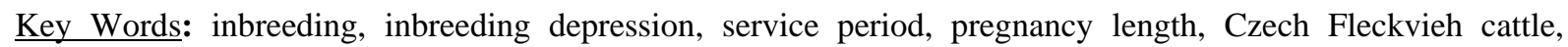
Holstein cattle

\section{Zusammenfassung}

Titel des Arbeit: Der Einfluss von Inzucht auf die Länge der Serviceperiode und die Trächtigkeitsdauer bei Kühen der Rassen Holstein und Tschechisches Fleckvieh nach der ersten Kalbung

Bei Erstlingskühen der Rassen Tschechisches Fleckvieh und Holstein wurde die Wirkung des Inzuchtgrades auf die Merkmale „Länge der Serviceperiode“ und „Trächtigkeitsdauer“ untersucht. Der Inzuchtgrad variierte zwischen 1,25 und 25\%. Die Länge der Serviceperiode und die Trächtigkeitsdauer der Inzuchtkühe wurden mit denen ihrer Nichtinzucht-Halbschwestern verglichen $(\mathrm{n}=1824)$. Bei den Halbschwestern handelte es sich um Zeit- und Stallgefährtinnen.

Es wurden 631810 Kühe, die in den Jahren 1985-2004 geboren wurden, erfasst. Die Inzuchtkühe wurden nach dem Inzuchtkoeffizienten $\left(F_{x}\right)$ in vier Gruppen geteilt (1,5-2,3\%; 3,0-5,0\%; 8,0-12,5\%; 25\%). Die Auswertung erfolgte mit dem Programm PROC GLM of SAS ${ }^{\circledR}$. Fixe Effekte waren die Rasse, der Zuchtwert des Vaters für Milchleistung, der Zuchtwert der Kuh, das Geburtsjahr und das Alter bei der ersten Kalbung.

Im Ergebnis der Untersuchungen hatten Inzuchtkühe eine verlängerte Serviceperiode, die mit Erhöhung des Inzuchtgrades stieg $(+2,81 ;+3,35 ;+4,53 ;+8,23$ Tage). Die Differenz zwischen Inzucht- und NichtinzuchtKühen betrug bei der Trächtigkeitsdauer $+0,3 ;+0,32 ;-0,08$ und 1,68 Tage. Sie war jedoch nicht statistisch gesichert. Eine Erhöhung des Inzuchtkoeffizienten um 1\% (Regressionskoeffizient) ergab eine Verlängerung der Serviceperiode und der Trächtigkeitsdauer von 0,22 und 0,063 Tagen. Die Zwischenkalbezeit (zwischen der ersten und zweiten Laktation) wurde mehr von der Länge der Serviceperiode beeinflusst als von der Trächtigkeitsdauer.

Schlüsselwörter: Inzucht, Inzuchtdepression, Serviceperiode, Trächtigkeitsdauer, Kühe der Rasse Tschechisches Fleckvieh, Kühe der Rasse Holstein 


\section{Introduction}

Inbreeding is consider one of the most important factors affecting the genetic structure and performance of breeding population (BIEDERMANN et al. 2003, 2004) Inbreeding has been studied as a specific question in small populations (KALLWEIT and BAULAIN, 2001; WOKAC, 2003; KHAN et al., 2007) and a general problem of breeding work (FREYER et al., 2005; PIRCHNER, 2004). SMITH et al. (1998) stated that the increasing of inbreeding coefficient $\left(F_{x}\right)$ per $1 \%$ increased age at the first calving by +0.55 , prolonged the first calving interval by $+0.31 \mathrm{~d}$ and reduced the productive life by 6 days. CASSELL et al. (2003) found out in Holstein livestock in the USA that the $F_{x}$ increasing of $1 \%$ extended service period of $0.15 \mathrm{~d}$. However, this increase was evaluated as non-significant. HERMAS et al (1987) studied impacts of different inbreeding levels within the interval $F_{x} 0-25.3 \%$. This experiment was carried out in Guersey cattle. The authors concluded that due to the inbreeding increase of $1 \%$ service period elongated of 2.3 day. Deterioration of reproduction traits was traced by WALL et al. (2005), who mentioned the increase of the calving interval of 2.8 day at $10 \%$ inbreeding level. The impact of inbreeding on reproduction traits in Slovak Fleckvieh livestock has been studied by BELLÉR et al. (1974). $F_{x}$ coefficient level was $12.5 \%$. The experimental and control group achieved the same values for the age at the first calving. Beside, the length of the calving interval in inbred animals was shorter (423 days) than in outbred cows (459 days). MIKŠÍK et al. (1978) calculated a significant evidential gravidity decrease of the pregnancy length after the first insemination with inbred mate, where the pregnancy dropped of $6.9 \%$. However, there was no difference in insemination index between inbred and outbred cows. Beside, the authors draw attention to the fact that fertility is considerably affected by different external factors. AHMAD et al. (1973) advert to non-significant shortening of reproduction traits. Authors calculated decrease in the age at first calving (1.2 d) and shortening of the first calving interval $(1.1 \mathrm{~d})$ with the increase $F_{X}$ of $1 \%$. THOMPSON et al. (2000 a, 2000 b) evaluated the impact of inbreeding in Holstein and Jersey livestock. Inbred cows with $\mathrm{F}_{\mathrm{x}}>10 \%$ showed increasing in the age at first calving of 25 days in Jerseys and 26 days in Holsteins. Female fertility in Holsteins measured by length of service period studied HOESCHELE (1991). She evaluated the service period prolongation of $2.6 \mathrm{~d}$ for $\mathrm{F}_{\mathrm{x}}=25 \%$. Some of the above mentioned authors assess service period and pregnancy length as one trait - the calving interval. The aim of this work was to evaluate the impact of inbreeding level on service period and pregnancy length in cows after the first calving separately. This method allows a more analytical consideration of performance.

\section{Materials and methods}

In this work, data included cows calved in years 1985-2004 at farms in the Czech Republic. Processing of breeding values data and monitoring of reproduction traits was finished toward June 2005. Calculations were performed on 631810 Holstein and Czech Fleckvieh cows. For the proper comparison each inbred cow (the range of $F_{x}$ coefficient 1.5-25\%) was assigned to at least one outbred equal (1.824 equals in sum). Inbred cows with their outbred equals were matched on characteristics such as (1) identical father, (2) first calving interval occurs in the same farm and (3) first calving happened in the same year and period ( \pm 5 months). Inbred cows and their matched 
outbred equals were subsequently divided according to the inbreeding coefficient of inbred cows into four groups $\left(F_{x}=1.5-2.3 \%, F_{x}=3.0-5.0 \%, F_{x}=8.0-12.5 \%, F_{x}=\right.$ $25.0 \%)$. The level of inbreeding - inbreeding coefficient $F_{x}$ was calculated as follows (WRIGHT, 1922)

$F_{x}=\Sigma 0.5^{n+n+1}\left(1+F_{a}\right)$

$\Sigma=$ sum over all path through to common ancestor

$\mathrm{n}=$ the number of generations from the sire to the common ancestor

$\mathrm{n}^{\prime}=$ the number of generations from the dam to the common ancestor

$\mathrm{F}_{\mathrm{a}}=$ the inbreeding coefficient of the common ancestor

Data were analysed using PROC GLM of SAS ${ }^{\circledR}$. The effects of inbreeding and other factors were estimated from the model as follows:

$Y_{i j k l m n}=\mu+I_{i}+B_{j}+B V S_{k}+B V M_{l}+C Y_{m}+\mathrm{CAg}_{\mathrm{n}}+e_{i j k l m n}$

$\mathrm{Y}_{\mathrm{ijklmn}}=$ corrected value of the reproduction trait

$\mu=$ mean value

$\mathrm{I}=\mathrm{F}_{\mathrm{x}}$ coefficient level (1=25.0 \%; 2=8.0-12.5 \%; 3=3.0-5.0 \%; 4=1.5-2.3 \%)

$\mathrm{B}=$ breed (Holstein cattle; Czech Fleckvieh cattle)

BVS = breeding value of the sire for milk production $(\leq 400 ; 401-800 ; 801 \geq)$

$\mathrm{BVM}=$ breeding value of the monitored individual for milk production $(\leq 1$; $1-250$;

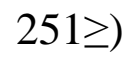

CY = year of calving (till year 1997; 1998-2000; 2001-2004)

$\mathrm{CA}=$ age at first calving /in months/ $(\leq 25.0 ; 25.1-27.0 ; 27.1 \geq)$

$\mathrm{e}=$ residual error.

Calculations were processed for inbred cows and outbred equals separately. A following comparison of average values between outbred and inbred groups of the same $\mathrm{F}_{\mathrm{x}}$ level has been made.

Results and Discussion

Variability in service period across the used effects and groups of inbred and outbred cows is mentioned in Tables 1 and 2.

There is obvious the significant difference between Holstein and Czech Fleckvieh in the length of service period. Similarly, the length of service period differs among groups defined by breeding values for milk production of sire or monitored individual. Some of these differences were significant on $\mathrm{p} \leq 0.01$. It corresponds to generally known correlations, where the increase of milk efficiency leads to deterioration in reproductive performance. There is an evident increase of the service period for inbred animals in comparison of the outbred ones (Tab. 3). 
Table 1

Variability in service period of inbred cows after first calving- allocated into groups according to the level of inbreeding - for each model effect separately (days) (Variabilität der Länge der Serviceperiode der Inzuchtkühe nach erster Kalbung - gegliedert nach Inzuchtgrad und Untersuchungsmodellen)

\begin{tabular}{|c|c|c|c|c|c|c|}
\hline Effects & & $\begin{array}{c}\mathrm{F}_{\mathrm{x}}=1,5-2,3 \% \\
\mathrm{LSM} \pm \mathrm{SE}\end{array}$ & $\begin{array}{c}\mathrm{F}_{\mathrm{x}}=3,0-5,0 \% \\
\mathrm{LSM} \pm \mathrm{SE}\end{array}$ & $\begin{array}{c}\mathrm{F}_{\mathrm{x}}=8,0-12,5 \% \\
\mathrm{LSM} \pm \mathrm{SE}\end{array}$ & $\begin{array}{c}\mathrm{F}_{\mathrm{x}}=25,0 \% \\
\mathrm{LSM} \pm \mathrm{SE}\end{array}$ & $\begin{array}{l}\text { All animals } \\
\mathrm{LSM} \pm \mathrm{SE}\end{array}$ \\
\hline \multirow{5}{*}{$\mathrm{F}_{\mathrm{x}}$} & 1 & & & & & $126,16 \pm 6,89$ \\
\hline & 2 & & & & & $116,06 \pm 6,93$ \\
\hline & 3 & & & & & $119,91 \pm 3,81$ \\
\hline & 4 & & & & & $117,03 \pm 5,92$ \\
\hline & $\mathrm{p}$ & & & & & n.s. \\
\hline \multirow{3}{*}{ Breed } & 1 & $126,67 \pm 5,65$ & $131,80 \pm 4,71$ & $133,94 \pm 10,24$ & $126,62 \pm 10,33$ & $131,02 \pm 3,42$ \\
\hline & 2 & $76,63 \pm 20,74$ & $105,63 \pm 7,99$ & $94,39 \pm 14,88$ & $130,21 \pm 12,01$ & $108,56 \pm 5,66$ \\
\hline & $\mathrm{p}$ & $1-2 *$ & $1-2 *$ & $1-2 *$ & n.s. & $1-2 *$ \\
\hline \multirow{4}{*}{ BVS } & 1 & $93,41 \pm 13,20$ & $103,60 \pm 8,03$ & $94,37 \pm 15,70$ & $114,28 \pm 13,53$ & $106,77 \pm 5,13$ \\
\hline & 2 & $102,35 \pm 14,15$ & $114,47 \pm 7,20$ & $118,64 \pm 14,20$ & $131,46 \pm 13,84$ & $119,71 \pm 5,31$ \\
\hline & 3 & $109,20 \pm 13,82$ & $138,08 \pm 7,46$ & $129,47 \pm 15,13$ & $139,51 \pm 16,45$ & $132,89 \pm 5,45$ \\
\hline & $\mathrm{p}$ & n.s. & $1-3 * * 2-3 * *$ & n.s. & n.s. & $1-3^{* *} 2-3 * *$ \\
\hline \multirow{4}{*}{ BVM } & 1 & $94,71 \pm 14,302$ & $118,40 \pm 7,05$ & $127,71 \pm 16,16$ & $115,95 \pm 12,08$ & $117,00 \pm 5,10$ \\
\hline & 2 & $85,84 \pm 12,72$ & $108,62 \pm 6,92$ & $106,62 \pm 14,49$ & $141,93 \pm 12,80$ & $112,51 \pm 4,92$ \\
\hline & 3 & $124,41 \pm 13,78$ & $129,11 \pm 6,54$ & $108,16 \pm 13,99$ & $127,38 \pm 18,03$ & $129,86 \pm 5,10$ \\
\hline & $\mathrm{p}$ & $2-3^{* *}$ & $2-3 *$ & n.s. & n.s. & $2-3 * *$ \\
\hline \multirow{4}{*}{ CY } & 1 & $94,80 \pm 13,60$ & $125,83 \pm 6,79$ & $119,96 \pm 12,34$ & $119,58 \pm 10,20$ & $119,22 \pm 4,56$ \\
\hline & 2 & $118,00 \pm 12,00$ & $116,51 \pm 5,74$ & $105,84 \pm 15,76$ & $127,89 \pm 18,76$ & $121,51 \pm 4,87$ \\
\hline & 3 & $92,16 \pm 14,91$ & $113,80 \pm 7,50$ & $117,08 \pm 15,00$ & $137,77 \pm 15,69$ & $118,64 \pm 5,57$ \\
\hline & $\mathrm{p}$ & n.s. & n.s. & n.s. & n.s. & n.s. \\
\hline \multirow{5}{*}{ CA } & 1 & $94,19 \pm 14,36$ & $110,50 \pm 7,17$ & $110,72 \pm 13,77$ & $113,79 \pm 19,15$ & $111,78 \pm 5,38$ \\
\hline & 2 & $109,58 \pm 13,33$ & $112,28 \pm 6,59$ & $110,58 \pm 15,91$ & $130,86 \pm 11,99$ & $118,87 \pm 4,93$ \\
\hline & 3 & $110,59 \pm 13,62$ & $123,20 \pm 6,86$ & $109,19 \pm 16,22$ & $126,05 \pm 13,86$ & $121,99 \pm 5,36$ \\
\hline & 4 & $92,25 \pm 16,06$ & $128,87 \pm 8,72$ & $126,17 \pm 17,60$ & $142,96 \pm 14,14$ & $126,52 \pm 6,04$ \\
\hline & $\mathrm{p}$ & n.s. & n.s. & n.s. & n.s. & n.s. \\
\hline
\end{tabular}

${ }^{*} \mathrm{p} \leq 0.05 ;{ }^{* *} \mathrm{p} \leq 0.01 ;$ n.s. $=$ non significant

Table 2

Variability in service period of outbred cows after first calving- allocated into groups according to the level of inbreeding of their inbred equals - for each model effect separately (days) (Variabilität der Länge der Serviceperiode der Nicht-Inzuchtkühe nach erster Kalbung - gegliedert nachUntersuchungsmodellen)

\begin{tabular}{|c|c|c|c|c|c|c|}
\hline Effects & & $\begin{array}{c}F_{x}=1,5-2,3 \% \\
L S M \pm S E\end{array}$ & $\begin{array}{c}\mathrm{F}_{\mathrm{X}}=3,0-5,0 \% \\
\mathrm{LSM} \pm \mathrm{SE}\end{array}$ & $\begin{array}{c}F_{x}=8,0-12,5 \% \\
L S M \pm S E\end{array}$ & $\begin{array}{l}\mathrm{F}_{\mathrm{x}} \quad 25,0 \% \\
\mathrm{LSM} \pm \mathrm{SE}\end{array}$ & $\begin{array}{l}\text { All animals } \\
\mathrm{LSM} \pm \mathrm{SE}\end{array}$ \\
\hline \multirow{3}{*}{ Breed } & 1 & $124,33 \pm 4,64$ & $124,60 \pm 4,03$ & $116,41 \pm 7,67$ & $113,33 \pm 7,58$ & $121,99 \pm 2,47$ \\
\hline & 2 & $100,98 \pm 16,58$ & $119,28 \pm 6,64$ & $101,12 \pm 11,31$ & $104,86 \pm 8,39$ & $114,17 \pm 4,31$ \\
\hline & $\mathrm{p}$ & n.s. & n.s. & n.s. & n.s. & n.s. \\
\hline \multirow{4}{*}{ BVS } & 1 & $108,15 \pm 10,63$ & $114,33 \pm 6,61$ & $117,01 \pm 10,51$ & $107,51 \pm 9,68$ & $113,81 \pm 4,03$ \\
\hline & 2 & $104,98 \pm 11,24$ & $122,48 \pm 5,98$ & $104,91 \pm 10,21$ & $104,13 \pm 9,99$ & $115,34 \pm 3,99$ \\
\hline & 3 & $124,84 \pm 11,99$ & $129,01 \pm 6,21$ & $104,38 \pm 11,97$ & $115,65 \pm 11,83$ & $125,10 \pm 4,27$ \\
\hline & $\mathrm{p}$ & n.s. & n.s. & n.s. & n.s. & n.s. \\
\hline \multirow{4}{*}{ BVM } & 1 & $99,08 \pm 11,94$ & $118,38 \pm 5,77$ & $102,84 \pm 12,35$ & $97,42 \pm 9,54$ & $109,68 \pm 4,06$ \\
\hline & 2 & $114,66 \pm 10,24$ & $112,79 \pm 5,51$ & $87,17 \pm 11,53$ & $114,70 \pm 7,36$ & $113,16 \pm 3,59$ \\
\hline & 3 & $124,23 \pm 11,20$ & $134,64 \pm 5,62$ & $136,28 \pm 9,70$ & $115,16 \pm 13,64$ & $131,40 \pm 3,96$ \\
\hline & $\mathrm{p}$ & & $1-3 * 2-3 * *$ & $1-3 * 2-3 * *$ & & $1-3 * * 2-3 * *$ \\
\hline \multirow{4}{*}{ CY } & 1 & $104,49 \pm 10,86$ & $124,18 \pm 5,33$ & $129,37 \pm 9,82$ & $111,02 \pm 8,15$ & $120,59 \pm 3,48$ \\
\hline & 2 & $115,81 \pm 9,57$ & $121,08 \pm 4,80$ & $118,38 \pm 10,75$ & $122,81 \pm 12,34$ & $120,73 \pm 3,49$ \\
\hline & 3 & $117,68 \pm 12,11$ & $120,55 \pm 6,33$ & $78,55 \pm 11,06$ & $93,44 \pm 11,90$ & $112,93 \pm 4,35$ \\
\hline & $\mathrm{p}$ & n.s. & n.s. & $1-3 * * 2-3 * *$ & n.s. & n.s. \\
\hline \multirow{5}{*}{ CA } & 1 & $123,95 \pm 11,89$ & $125,49 \pm 6,40$ & $116,41 \pm 11,35$ & $113,08 \pm 11,84$ & $124,12 \pm 4,42$ \\
\hline & 2 & $108,06 \pm 10,07$ & $122,21 \pm 5,05$ & $109,80 \pm 11,69$ & $120,39 \pm 8,93$ & $119,75 \pm 3,52$ \\
\hline & 3 & $116,74 \pm 10,63$ & $119,07 \pm 5,69$ & $111,64 \pm 11,15$ & $111,46 \pm 10,76$ & $117,43 \pm 3,93$ \\
\hline & 4 & $101,87 \pm 13,83$ & $120,98 \pm 7,93$ & $97,20 \pm 12,76$ & $91,44 \pm 12,25$ & $111,02 \pm 5,16$ \\
\hline & $\mathrm{p}$ & n.s. & n.s. & n.s. & $2-4^{*}$ & $1-4 *$ \\
\hline
\end{tabular}

${ }^{*} \mathrm{p} \leq 0.05 ; * * \mathrm{p} \leq 0.01 ;$ n.s. $=$ non significant 
Table 3

Service period for inbred and outbred cows after first calving (days) (Länge der Serviceperiode der Inzucht- und Nichtinzucht-Kühe nach erster Kalbung)

\begin{tabular}{ccccccc}
\hline & $\mathrm{F}_{\mathrm{x}}(\%)$ & $1,5-2,3$ & $3,0-5,0$ & $8,0-12,5$ & 25,0 & all animals \\
\hline \multirow{4}{*}{ Inbred cows } & $\mathrm{n}$ & 138 & 340 & 81 & 89 & 648 \\
& $\mathrm{Y}_{\mathrm{ijk} k \mathrm{mn}}$ & 126,94 & 130,69 & 122,47 & 119,56 & 127,34 \\
& $\mathrm{R}^{2}$ & 0,1791 & 0,1383 & 0,1273 & 0,1173 & 0,1034 \\
& $\mathrm{~V}_{\mathrm{x}}$ & 47,23 & 46,47 & 52,34 & 48,78 & 47,67 \\
\hline \multirow{4}{*}{ Outbred equals } & $\mathrm{n}$ & 138 & 340 & 81 & 89 & 648 \\
& $\mathrm{Y}_{\mathrm{ijkklmn}}$ & 124,13 & 127,34 & 117,94 & 111,33 & 123,28 \\
& $\mathrm{R}^{2}$ & 0,1833 & 0,084 & 0,2312 & 0,1482 & 0,0954 \\
& $\mathrm{~V}_{\mathrm{x}}$ & 38,23 & 38,69 & 39,05 & 36,5 & 38,51 \\
\hline \multicolumn{2}{c}{ Differences (inbred - outbred) } & $\mathbf{2 , 8 1}$ & $\mathbf{3 , 3 5}$ & $\mathbf{4 , 5 3}$ & $\mathbf{8 , 2 3}$ & $\mathbf{4 , 0 6}$ \\
\hline
\end{tabular}

This change is represented in Figure 1. The lowest increase was recorded for the lowest $\mathrm{F}_{\mathrm{x}}$ coefficients (+2.81 day) and the highest difference between inbred and outbred cows was at $\mathrm{Fx}=25 \%$ (+8.23 day). However, this increase of service period was not significant. The mentioned differences correspond to the subsequently calculated values of correlation and regression coefficients. There was assessed a very low positive correlation between Fx and differences in service period; $r=0.023$. The regression coefficient was byx $=0.22$ day (where $\mathrm{x}=$ coefficient $\mathrm{Fx}$ in $\%, \mathrm{y}=$ difference in service period between inbred and outbred cows - in days). The Fx coefficient increasing by $1 \%$ extends the service period by 0.22 day.

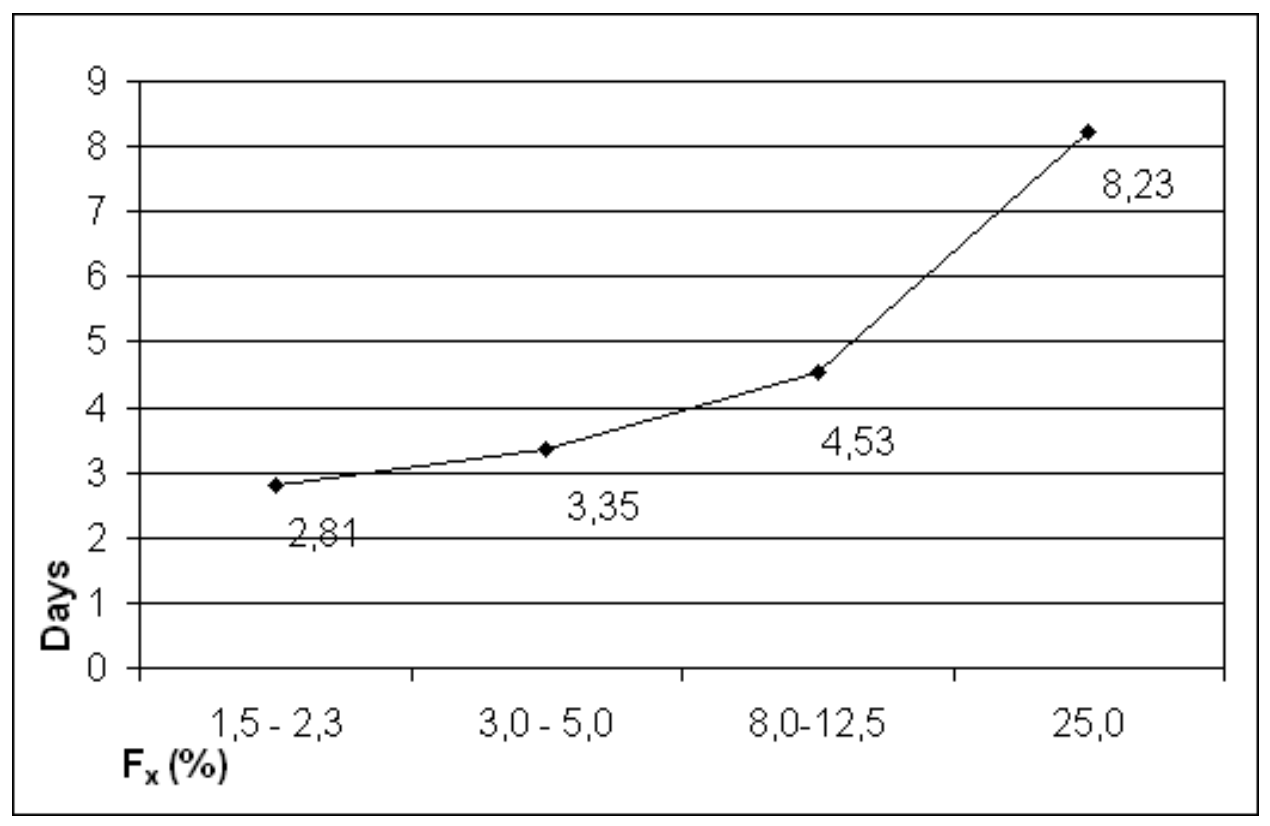

Fig. 1: Effect of inbreeding on service period. Differences in service period between inbred and outbred cows after first calving (days) (Inzuchteffekte bei der Länge der Serviceperiode. Unterschiede in der Länge der Serviceperiode zwischen den Inzucht- und Nichtinzucht-Kühen nach erster Kalbung)

Variability of pregnancy length across the used effects and groups of inbred and outbred cows is mentioned in Tables 4 and 5. There are obvious the significant differences between Holsteins and Czech Fleckviehs in the length of pregnancy. However, the other effects, including Fx levels are not significant. 
Table 4

Variability in pregnancy length of inbred cows after first calving- allocated into groups according to the level of inbreeding - for each model effect separately (days) (Variabilität in der Trächtigkeitsdauer bei Inzuchtkühen nach erster Kalbung - gegliedert nach Inzuchtgrad und Untersuchungsmodellen)

\begin{tabular}{|c|c|c|c|c|c|c|}
\hline Effects & & $\begin{array}{c}F_{x}=1,5-2,3 \% \\
L S M \pm S E\end{array}$ & $\begin{array}{c}F_{x}=3,0-5,0 \% \\
L S M \pm S E\end{array}$ & $\begin{array}{c}\mathrm{F}_{\mathrm{x}}=8,0-12,5 \% \\
\mathrm{LSM} \pm \mathrm{SE}\end{array}$ & $\begin{array}{c}\mathrm{F}_{\mathrm{x}}=25,0 \% \\
\mathrm{LSM} \pm \mathrm{SE}\end{array}$ & $\begin{array}{l}\text { All animals } \\
\mathrm{LSM} \pm \mathrm{SE}\end{array}$ \\
\hline \multirow{5}{*}{$F_{x}$} & 1 & & & & & $283,99 \pm 0,69$ \\
\hline & 2 & & & & & $283,37 \pm 0,64$ \\
\hline & 3 & & & & & $282,77 \pm 0,37$ \\
\hline & 4 & & & & & $282,80 \pm 0,60$ \\
\hline & $\mathrm{p}$ & & & & & n.s. \\
\hline \multirow{3}{*}{ Breed } & 1 & $279,88 \pm 0,55$ & $280,23 \pm 0,42$ & $280,35 \pm 0,79$ & $280,76 \pm 1,12$ & $280,56 \pm 0,33$ \\
\hline & 2 & $285,28 \pm 2,01$ & $285,44 \pm 0,77$ & $285,20 \pm 1,17$ & $286,21 \pm 1,26$ & $285,91 \pm 0,55$ \\
\hline & $\mathrm{p}$ & $1-2 * *$ & $1-2 * *$ & $1-2 * *$ & $1-2 * *$ & $1-2 * *$ \\
\hline \multirow{4}{*}{ BVS } & 1 & $281,50 \pm 1,24$ & $283,09 \pm 0,61$ & $282,20 \pm 1,12$ & $282,38 \pm 1,50$ & $283,33 \pm 0,44$ \\
\hline & 2 & $283,49 \pm 1,42$ & $282,43 \pm 0,73$ & $284,07 \pm 1,15$ & $284,57 \pm 1,58$ & $282,98 \pm 0,55$ \\
\hline & 3 & $282,75 \pm 1,26$ & $283,00 \pm 0,69$ & $282,04 \pm 1,30$ & $283,51 \pm 1,61$ & $283,38 \pm 0,50$ \\
\hline & p & n.s. & n.s. & n.s. & n.s. & n.s. \\
\hline \multirow{4}{*}{ BVM } & 1 & $281,76 \pm 1,34$ & $283,49 \pm 0,65$ & $281,16 \pm 1,34$ & $284,27 \pm 1,30$ & $283,37 \pm 0,49$ \\
\hline & 2 & $282,64 \pm 1,23$ & $282,52 \pm 0,71$ & $282,71 \pm 1,38$ & $284,10 \pm 1,41$ & $283,06 \pm 0,48$ \\
\hline & 3 & $283,35 \pm 1,30$ & $282,50 \pm 0,62$ & $284,44 \pm 1,01$ & $282,09 \pm 1,90$ & $283,27 \pm 0,48$ \\
\hline & $\mathrm{p}$ & n.s. & n.s. & n.s. & n.s. & n.s. \\
\hline \multirow{4}{*}{ CY } & 1 & $283,64 \pm 1,28$ & $284,04 \pm 0,65$ & $283,56 \pm 0,97$ & $285,38 \pm 1,03$ & $284,40 \pm 0,41$ \\
\hline & 2 & $281,84 \pm 1,26$ & $282,26 \pm 0,58$ & $280,99 \pm 1,34$ & $280,91 \pm 2,02$ & $282,61 \pm 0,50$ \\
\hline & 3 & $282,27 \pm 1,39$ & $282,22 \pm 0,70$ & $283,76 \pm 1,30$ & $284,17 \pm 1,78$ & $282,69 \pm 0,53$ \\
\hline & p & n.s. & $1-2 * 1-3 *$ & n.s. & $1-2 *$ & $1-2 * * 1-3 * *$ \\
\hline \multirow{5}{*}{ CA } & 1 & $281,62 \pm 1,38$ & $283,14 \pm 0,69$ & $282,19 \pm 1,42$ & $282,71 \pm 1,98$ & $282,87 \pm 0,53$ \\
\hline & 2 & $284,43 \pm 1,27$ & $282,40 \pm 0,64$ & $281,70 \pm 1,39$ & $284,87 \pm 1,23$ & $283,62 \pm 0,47$ \\
\hline & 3 & $282,46 \pm 1,38$ & $282,09 \pm 0,64$ & $284,46 \pm 0,99$ & $281,92 \pm 1,47$ & $282,69 \pm 0,52$ \\
\hline & 4 & $281,81 \pm 1,54$ & $283,71 \pm 0,87$ & $282,72 \pm 1,36$ & $284,46 \pm 1,63$ & $283,74 \pm 0,60$ \\
\hline & p & $1-2 *$ & n.s. & n.s. & n.s. & n.s \\
\hline
\end{tabular}

Table 5

Variability in pregnancy of outbred cows after first calving- allocated into groups according to the level of inbreeding of their inbred equals - for each model effect separately (days) (Variabilität bei der Trächtigkeitsdauer der Nichtinzucht-Kühe nach erster Kalbung - gegliedert nach dem Inzuchtgrad der Halbgeschwister der Inzuchtkühe und Untersuchungsmodellen)

\begin{tabular}{|c|c|c|c|c|c|c|}
\hline Effects & & $\begin{array}{c}\mathrm{F}_{\mathrm{x}}=1,5-2,3 \% \\
\mathrm{LSM} \pm \mathrm{SE}\end{array}$ & $\begin{array}{c}\mathrm{F}_{\mathrm{x}}=3,0-5,0 \% \\
\mathrm{LSM} \pm \mathrm{SE}\end{array}$ & $\begin{array}{c}\mathrm{F}_{\mathrm{x}}=8,0-12,5 \% \\
\mathrm{LSM} \pm \mathrm{SE}\end{array}$ & $\begin{array}{c}\mathrm{F}_{\mathrm{x}}=25,0 \% \\
\mathrm{LSM} \pm \mathrm{SE}\end{array}$ & $\begin{array}{l}\text { All animals } \\
\mathrm{LSM} \pm \mathrm{SE}\end{array}$ \\
\hline \multirow{3}{*}{ Breed } & 1 & $279,93 \pm 0,46$ & $280,17 \pm 0,36$ & $280,35 \pm 0,79$ & $281,17 \pm 1,11$ & $280,27 \pm 0,25$ \\
\hline & 2 & $286,31 \pm 1,66$ & $285,33 \pm 0,65$ & $285,20 \pm 1,17$ & $282,94 \pm 1,23$ & $285,07 \pm 0,46$ \\
\hline & $\mathrm{p}$ & $1-2 * *$ & $1-2 * *$ & $1-2 *$ & n.s. & $1-2 * *$ \\
\hline \multirow{4}{*}{ BVS } & 1 & $284,18 \pm 1,08$ & $283,34 \pm 0,55$ & $282,20 \pm 1,12$ & $280,13 \pm 1,49$ & $282,88 \pm 0,40$ \\
\hline & 2 & $283,37 \pm 1,16$ & $283,17 \pm 0,62$ & $284,07 \pm 1,14$ & $284,31 \pm 1,62$ & $283,33 \pm 0,45$ \\
\hline & 3 & $281,81 \pm 1,21$ & $281,75 \pm 0,61$ & $282,04 \pm 1,30$ & $281,74 \pm 1,57$ & $281,80 \pm 0,45$ \\
\hline & $\mathrm{p}$ & n.s. & n.s. & $1-2^{*}$ & $1-2^{*}$ & $2-3^{*}$ \\
\hline \multirow{4}{*}{ BVN } & 1 & $282,04 \pm 1,23$ & $282,38 \pm 0,57$ & $281,16 \pm 1,34$ & $283,07 \pm 1,39$ & $282,08 \pm 0,43$ \\
\hline & 2 & $281,71 \pm 1,05$ & $283,36 \pm 0,55$ & $282,71 \pm 1,38$ & $283,68 \pm 1,21$ & $282,73 \pm 0,39$ \\
\hline & 3 & $285,61 \pm 1,15$ & $282,52 \pm 0,58$ & $284,44 \pm 1,01$ & $279,43 \pm 2,08$ & $283,20 \pm 0,44$ \\
\hline & $\mathrm{p}$ & $1-3 * 2-3 * *$ & n.s. & n.s. & n.s. & n.s. \\
\hline \multirow{4}{*}{$\mathrm{CY}$} & 1 & $284,35 \pm 1,06$ & $283,26 \pm 0,52$ & $283,56 \pm 0,97$ & $283,68 \pm 1,17$ & $283,41 \pm 0,36$ \\
\hline & 2 & $282,55 \pm 1,02$ & $282,73 \pm 0,49$ & $280,99 \pm 1,34$ & $281,94 \pm 1,87$ & $282,30 \pm 0,39$ \\
\hline & 3 & $282,47 \pm 1,19$ & $282,27 \pm 0,60$ & $283,76 \pm 1,30$ & $280,56 \pm 1,91$ & $282,29 \pm 0,45$ \\
\hline & $\mathrm{p}$ & n.s. & n.s. & n.s. & n.s. & $1-2 * 1-3 *$ \\
\hline \multirow{5}{*}{ CA } & 1 & $281,54 \pm 1,31$ & $282,91 \pm 0,69$ & $282,19 \pm 1,42$ & $280,11 \pm 1,67$ & $281,88 \pm 0,52$ \\
\hline & 2 & $283,01 \pm 0,97$ & $282,16 \pm 0,48$ & $281,70 \pm 1,39$ & $281,33 \pm 1,40$ & $282,13 \pm 0,36$ \\
\hline & 3 & $283,57 \pm 1,06$ & $282,37 \pm 0,52$ & $284,46 \pm 0,99$ & $283,31 \pm 1,66$ & $283,03 \pm 0,39$ \\
\hline & 4 & $284,36 \pm 1,35$ & $283,57 \pm 0,81$ & $282,72 \pm 1,36$ & $283,50 \pm 1,58$ & $283,64 \pm 0,55$ \\
\hline & $\mathrm{p}$ & n.s. & n.s. & n.s. & n.s. & $1-4 * 2-4 *$ \\
\hline
\end{tabular}


There is a small and non-significant increase in the pregnancy length for inbred animals in comparison with outbred equals (Tab. 6 and Fig. 2). The differences are higher for groups with a higher Fx. The highest difference $1.68 \mathrm{~d}$ is for Fx=25\% (nonsignificant). The mentioned differences correspond to the subsequently calculated values of correlation and regression coefficients. There was assessed a very low positive correlation between Fx and differences in pregnancy length $r=+0.0658$. The regression coefficient was $b_{\mathrm{yx}}=0.063$ day (where $\mathrm{x}=$ coefficient $\mathrm{Fx}$ in \%, $\mathrm{y}=$ difference in pregnancy length between inbred and outbred cows in days). The Fx coefficient increasing by $1 \%$ extends the pregnancy length by 0.063 day.

Table 6

Pregnancy length for inbred and outbred cows after first calving (days) (Trächtigkeitsdauer von Inzucht- und Nichtinzucht-Kühen nach erster Kalbung)

\begin{tabular}{ccccccc}
\hline & $\mathrm{F}_{\mathrm{x}}(\%)$ & $1,5-2,3$ & $3,0-5,0$ & $8,0-12,5$ & 25,0 & All animals \\
\hline \multirow{4}{*}{ Inbred cows } & $\mathrm{n}$ & 127 & 321 & 82 & 77 & 607 \\
& $\mathrm{Y}_{\mathrm{ijklmn}}$ & 280,45 & 281,1 & 282,82 & 284,75 & 281,66 \\
& $\mathrm{R}^{2}$ & 0,1436 & 0,1794 & 0,3091 & 0,3227 & 0,2091 \\
& $\mathrm{~V}_{\mathrm{x}}$ & 1,99 & 1,98 & 2,01 & 1,97 & 1,99 \\
\hline \multirow{4}{*}{ Outbred equals } & $\mathrm{n}$ & 127 & 321 & 82 & 77 & 607 \\
& $\mathrm{Y}_{\mathrm{ijklmn}}$ & 280,15 & 280,78 & 282,9 & 283,08 & 281,23 \\
& $\mathrm{R}^{2}$ & 0,2714 & 0,2686 & 0,296 & 0,2484 & 0,2341 \\
\hline Differences (inbred - outbred) & $\mathrm{V}_{\mathrm{x}}$ & 1,6 & 1,63 & 1,76 & 1,94 & 1,7 \\
\hline
\end{tabular}

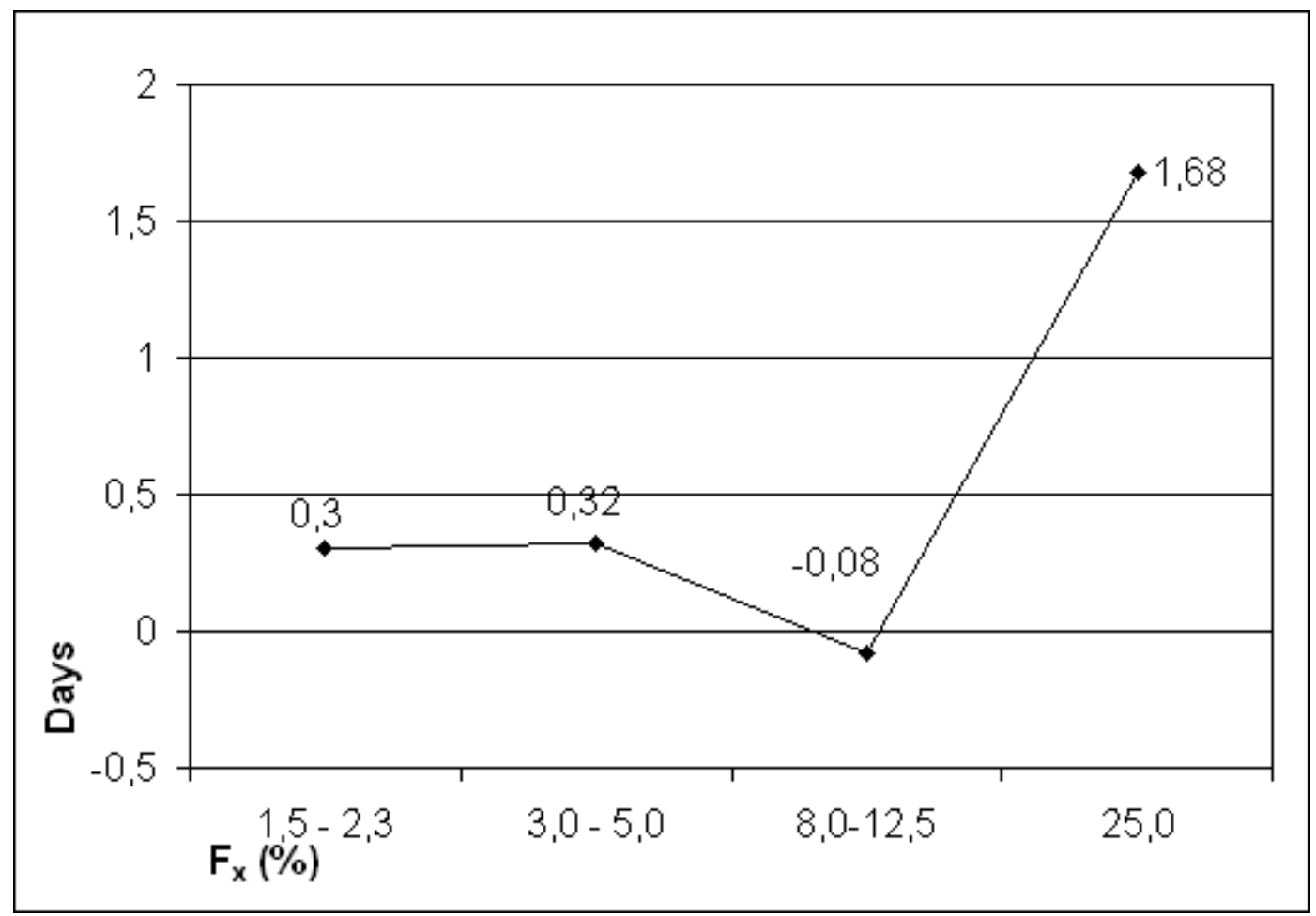

Fig. 2: Effect of inbreeding on pregnancy length. Differences in pregnancy length between inbred and outbred cows (days) (Inzuchteinfluss bei der Trächtigkeitsdauer. Unterschiede in der Trächtigkeitsdauer zwischen Inzucht- und Nichtinzucht-Kühen) 
The above-mentioned values of regression and correlation analyses of service period and pregnancy length are in accordance with the findings of other authors. Very similar results were published by CASSELL et al (2003), who mentioned that an increase of Fx coefficient by $1 \%$ corresponding to 0.15 day of inbred depression for service period. HOESCHELE (1991) indicated the increase of service period of 2.6 of the day for $\mathrm{Fx}=25.0 \%$. Partly higher regression calculated HERMAS et al. (1987), who mentioned increasing of service period by +1.4 day for $1 \%$ increasing of Fx. Anyway, also these authors evaluated increasing of service period for inbred animals as non-significant.

In this work, no significant depressive effects of inbreeding on service period and pregnancy length were detected. The increase in service period $(2.81 ; 3.35 ; 4.53$ and 8.23 day) and in pregnancy length (0.3; 0.32 ; -0.08 and 1.68 day) was recorded for inbred animal groups according to the levels of inbreeding coefficient (1.5-2.3\%; 3.0$5.0 \%$; 8.0-12.5 \% and $25.0 \%$, respectively according to values of traits). The level of inbreeding coefficient corresponds to the increase of service period (+4.06 day) and pregnancy length ( +0.43 day) for all animals. Estimates of depression per $1 \%$ of increase in inbreeding were $+0.22 \mathrm{~d}$ for service period and $+0.063 \mathrm{~d}$ for pregnancy length. There were assessed low positive correlations between depression and the reproductive traits. Service period and pregnancy length were affected positively by breeding value of the sire for milk production, breeding value of the monitored individual for milk production and breed. We have found out a higher variability (Vx) of service period and pregnancy length in inbred animals than in outbred ones. We can conclude that differences in length of the first calving interval between outbred and inbred cows are affected more by service period than pregnancy length.

\section{Acknowledgments}

This study was supported by the the Grant Agency of the Ministry of Agriculture of the Czech Republic, Project No. 1B44035 and the Ministry of Education, Youth and Sports of the Czech Republic (MSM 2678846201).

\section{References}

AHMAD, Z.; AHMAD, M.D.; QURESHI, A.W.:

Influence of Inbreeding on Performance Traits of Sahiwal Cattle, J. Dairy Sci. 57 (1973), 1225-1227

BELLER I.,; PLESNIK J. :

Pôsobenie príbuzenskej plemenitby na úžitkovost' dojníc. (Effect of inbreeding on performance of dairy cattle.) Živočišná výroba (Czech J.Anim.Sci.) 19 (1974), 117-124

BIEDERMANN, G.; WALDMANN, S.; MAUS, F.:

Genetische Analyse der Population des Hinterwälder Rindes (Genetic analysis of the population of Hinterwald cattle). Arch. Tierz., Dummerstorf 46 (2003) 4, 307-319

BIEDERMANN, G.; OTT, B.; RÜBESAM, K.; MAUS, F.:

Genetic analysis of the population of Vorderwald cattle. Arch. Tierz., Dummerstorf 47 (2004) 2, 141153

CASSELL, B.G.; ADAMEC, V.; PEARSON, R.E.:

Effect of Incomplete Pedigrees on Estimates of Inbreeding and Inbreeding Depression for Days to First Service and Summit Milk Yield in Holsteins and Jerseys, J. Dairy Sci., 86 (2003), 2967-2976

FREYER, G.; HERNANDEZ-SANCHES, J.; CASSELL, B. G.:

A note on inbreeding on. dairy cattle breeding. Arch. Tierz., Dummerstorf 48 (2005) 2, 130-137

HERMAS, S.A.; YOUNG, C.W.; RUST, J.W.: 
Effects of Mild Inbreeding on Productive and Reproductive Performance of Guernsey Cattle. J. Dairy Sci., 70 (1987), 712-714

HOESCHELE, I.:

Additive and Nonadditive Genetic Variance in Female Fertility of Holsteins, J. Dairy Sci., 74 (1991) 5, 1743-1751

KHAN, M.S.; ALI, A.; HYDER, A.U.; CHATTA, A.I.:

Effect of inbreeding on growth and reproduction traits of Beetal goats. Arch. Tierz., Dummerstorf $\mathbf{5 0}$ (2007), 197-203

KALLWEIT, E.; BAULAIN, U.:

Reproduction performance and degree of inbreeding in a small Finnsheep population during a 34-year period. Arch. Tierz., Dummerstorf 44 (2001), 263-270

MIKŠÍK, J.; KADEČKA, J.; UHLÍŘ, B.:

Vliv inbreedingu na reprodukci u českého strakatého skotu (Effect of inbreeding on reproduction in Czech Fleckvieh cattle), Živočišná výroba (Czech J.Anim.Sci.), 23 (1978), 129 -135

SAS 8.2 - GLM procedure Copyright c 1999-2007 by SAS Institute Inc., Cary, NC, USA

SMITH, L.A.; CASSELL, B.G.; PEARSON, R.E.:

The Effects of Inbreeding on the Lifetime Performance of Dairy Cattle, J. Dairy Sci., 81 (1998), 27292737

PIRCHNER, F.:

Use of inbred sires to exploit epistatic variance. Arch. Tierz., Dummerstorf 47 (2004) 6, 605-608

THOMPSON, J.R.; EVERET, R.W.; WOLFE, C.W.:

Effects of Inbreeding on Production and Survival in Jerseys. J. Dairy Sci., 8 (2000a), 2131-2137

THOMPSON, J.R.; EVERET, R.W.; HAMMERSCHMIDT, N.L.:

Effects of Inbreeding on Production and Survival in Holsteins. J. Dairy Sci., 83 (2000b), 1856-1864

WALL, E.; BROTHERSTONE, S.; KEARNEY, J. F.; WOOLLIAMS, J. A.; COFFEY, M. P.:

Impact of Nonadditive Genetic Effects in the Estimation of Breeding Values for Fertility and Correlated Traits, J. Dairy Sci., 88 (2005), 376-385

WOKAC, R. M.:

Bedeutung der Inzucht bei Tauernschecken-Ziegen (On the importance of inbreeding at Tauernschecken goats), Arch. Tierz., Dummerstorf 46 (2003) 5, 455-470

WRIGHT, S.:

Coefficients of inbreeding and relationship. American Naturalist, 56 (1922), 330-338

Received: 2007-05-14

Accepted: 2007-07-02

Authors' addresses

Ing. JIŘÍ BEZDÍČEK*, Ph.D., MSc. ALEŠ DUFEK

Research Institute for Cattle Breeding, Ltd.,

Výzkumníků 267 Rapotín

78813 VIKÝŘOVICE, CZECH REPUBLIC

*Corresponding Author, E-Mail: jiri.bezdicek@vuchs.cz

Prof. Ing. JAN ŠUBRT, CSc., Ing. RADEK FILIPČĆK

Mendel University of Agriculture and Forestry Brno, Zemědělská 1665/1,

61300 BRNO, CZECH REPUBLIC

Ing. MAREK BJELKA, PhD.

Agroresearch Rapotín, Ltd.,

Výzkumníků 267 Rapotín

78813 VIKÝŘOVICE, CZECH REPUBLIC 